\title{
Lane Change Behavior on Freeways: An Online Survey Using Video Clips
}

\author{
V. L. Knoop $\mathbb{D}^{1},{ }^{1}$ M. Keyvan-Ekbatani, ${ }^{2}$ M. de Baat, ${ }^{1}$ H. Taale $\mathbb{C}^{1},{ }^{1,3}$ and S. P. Hoogendoorn ${ }^{1}{ }^{1}$ \\ ${ }^{1}$ Delft University of Technology, Netherlands \\ ${ }^{2}$ University of Canterbury, New Zealand \\ ${ }^{3}$ Rijkswaterstaat and Delft University of Technology, Netherlands \\ Correspondence should be addressed to V. L. Knoop; v.l.knoop@tudelft.nl
}

Received 2 November 2017; Revised 9 March 2018; Accepted 7 May 2018; Published 24 June 2018

Academic Editor: Jose E. Naranjo

Copyright (C) 2018 V. L. Knoop et al. This is an open access article distributed under the Creative Commons Attribution License, which permits unrestricted use, distribution, and reproduction in any medium, provided the original work is properly cited.

\begin{abstract}
Freeways form an important part of the road network. Yet, driving behavior on freeways, in particular lane changes and the relation with the choice of speed, is not well understood. To overcome this, an online survey has been carried out. Drivers were shown video clips, and after each clip they had to indicate what they would do after the moment the video stopped. A total of 1258 Dutch respondents completed the survey. The results show that most people have a strategy to choose a speed first and stick to that, which is the first strategy. A second, less often chosen, strategy is to choose a desired lane and adapt the speed based on the chosen lane. A third strategy, slightly less frequently chosen, is that drivers have a desired speed, but contrary to the first strategy, they increase this speed when they are in a different lane overtaking another driver. A small fraction have neither a desired speed nor a desired lane. Of the respondents $80 \%$ use the right lane if possible, and $80 \%$ avoid overtaking at the right. Also $80 \%$ give way to merging traffic. The survey was validated by 25 survey respondents also driving an instrumented vehicle. The strategies in this drive were similar to those in the survey. The findings of this work can be implemented in traffic simulation models, e.g., to determine road capacity and constraints in geometric design.
\end{abstract}

\section{Introduction}

Freeways constitute a principal part of the transport infrastructure. However, if traffic exceeds capacity, it gets congested. Therefore, capacity is an important traffic variable. It is determined by the average minimum headway between two successive vehicles, and the lane they choose. In practice, the road is often underutilized since not all lanes are operating at capacity in high demand (e.g., Daganzo [1]; Knoop et al. [2]). This unequal lane distribution is a consequence of the lane change behavior of drivers.

Lane changes may cause disruptions and oscillations [3] and consequently influence the capacity of the road by leaving voids and reducing flow [4]. Also, oscillations can result into problems including safety hazards, extra fuel consumption, emissions, delays, and driver discomfort. To mitigate these problems, it would be good if the behavior of drivers was known, and the traffic dynamics could be predicted. This could be useful for road design or measures to improve driver behavior. In this paper, the lane choice of drivers is investigated under European conditions, which means keepright-unless-overtaking regulations. In a previous study [5] it was shown with a test with 10 drivers that various drivers had different strategies (summarized in Section 2.1). Using a large scale survey the research described in this paper quantifies further which part of the population uses which strategy.

The research objective is to get insights into the distribution of drivers over the different strategies. These insights can be used to better describe traffic operations in microscopic simulation models. To be able to use these models for (exante) assessing road design or traffic management measures, a proper model for lane change and lane choice behavior is crucial, which should be based on the insight of driving behavior. To obtain insights into this behavior, a large scale state preference survey among Dutch drivers was conducted. The research has been submitted and approved by the Ethics Committee of the Delft University of Technology. Preliminary results of the research have been presented 
earlier [6]. The main contributions of the work presented in the current paper are the use of video clips to introduce possible choices and the large response of this realistic stated preference survey, which gives confidence in the results.

Since the survey gives a stated preference which might differ from a revealed preference, the survey was (face) validated. To that end 25 respondents to the survey also participated in a drive on the road in an instrumented vehicle, recording drivers' actions. For practical reasons, the research started with the drives which were recorded on video. This gave footage of many different traffic situations. Clips thereof were utilized to introduce various questions in the online survey. Typical situations were reconstructed in an approximately ten-second video clip. Respondents were asked how they would cope with the situation which was shown in the video clip. Generally, that could involve a speed and/or a lane adaptation. For the 25 participants in the drive, an interview followed right after the drive. As validation for the stated preference survey, this group's choice in the drive and the outcomes of their interview were compared with their choices in the survey. As stated before, the main goal was to describe the driving behavior of Dutch drivers. It is possible that driving behavior differs for drivers in other countries. To check this, the same survey has been distributed in other countries.

The paper is structured as follows. First, Section 2 discusses the background for driving behavior and survey techniques. The survey setup is then presented in Section 3, followed by the results from the survey in Section 4. The validation of the survey using the instrumented vehicle drives is presented in Section 5. Section 6 shows the validity of the survey for different countries. In the remainder of the paper, the drive in the instrumented vehicle will be called drive. The word respondent will be used to denote respondents to the survey, whereas an participant is a person participating in the drive.

\section{Literature Review}

In this section, previous research on this topic is discussed from two points of view. First, it is important to know which aspects related to this topic deserve attention. To that end, the literature on lane change behavior on multilane freeways is studied in Section 2.1. Having found the relevant aspects, it is important to study them in the right way, because various techniques are available. Section 2.2 discusses advantages and disadvantages of particular ways of studying driving behavior.

2.1. Lane Change Behavior on Multilane Freeways. In order to quantify the complex features of multilane traffic, understanding lane change behavior on freeways is essential. As addressed by Toledo et al. [7] and Kesting et al. [8], modeling lane changing maneuvers is a key component of microscopic traffic simulation tools. In the vast majority of microscopic simulation models, a desired speed is chosen first, and the lane is being adapted if needed. Several studies have been carried out on developing realistic lane change models. However, despite its importance in mimicking traffic operations on freeways, among the researchers it did not attain as much attention as the car-following models (for a review see Moridpour et al. [9] and Zheng [10]). Rulebased and discrete-choice-based models are the most popular microscopic lane changing algorithms in literature [11]. In rule-based models, considering the heterogeneity among drivers, different gap acceptance conditions have been taken into account. It was argued that oscillations are the result of driver heterogeneous reactions to deceleration waves on freeways [12]. Many lane changing models incorporate the heterogeneity of drivers by different parameter sets. For the car-following behavior, it has been shown that there is variation of strategies among drivers [13]. A recent study [5] confirmed that drivers also have completely different strategies to choose lanes and that speed and lane changing choices are related. This shows that considering different parameter sets may not be a reliable approach to model heterogeneous driving behavior in a microscopic simulation environment, because it does not capture the driver choices realistically. The more detailed discrete-choice-based algorithms apply logit models to simulate driver behavior [14]. This group of microscopic algorithms can describe lane changing behavior in detail, but they often contain a large number of parameters which makes it hard to calibrate and validate the model [8]. The recently developed LMRS model [15] combines the route, speed, and keep right incentives for lane changing with the synchronization and relaxation of car-following behavior, but also in this model lane choice strategies are not considered.

As mentioned before, the study described by KeyvanEkbatani et al. [5] has led to a categorization of the lane change decision process (i.e., strategies), based on a drive with 10 participants and a series of interviews. Particularly, it has been revealed that people drive on the freeway according to the following four strategies.

Strategy 1 (speed leading). Drivers choose a desired speed for driving on a freeway stretch and try to stick to that speed as much as possible. If required, they change lanes and overtake to continue driving their speed. Also during the overtaking maneuver, they stick to their desired speed.

Strategy 2 (speed leading with speed increase at overtaking). As in the previous strategy, drivers choose a desired speed and try to maintain that speed as much as possible. They may change lane to overtake a slower driver. Different from the speed leading strategy, the drivers increase speed when they are overtaking and do not stick to the original desired speed. (Keyvan-Ekbatani et al. [5] call this strategy "speed leading with overtaking", which might lead to misunderstandings since overtaking is also possible in Strategy 1).

Strategy 3 (lane leading). Drivers choose a specific lane and adapt their speed to the speed of that lane, but within margins (typical accepted range is approximately $40 \mathrm{~km} / \mathrm{h}$ ).

Strategy 4 (traffic leading). Drivers have neither a specific lane nor a desired speed in mind and "go with the flow".

2.2. Studying Lane Change Behavior. Several approaches are available to study driving behavior on freeways. Our 
work focuses on lane changing, which, in general, is not studied that much. More often, merging, as a special case of lane changing, is studied. Therefore, we look into the methodologies used to study merging behavior, since they are potentially useful for lane changing in general. The following four approaches have been used to study freeway driving behavior: interviews or group discussions (focus groups), an instrumented vehicle, a driving simulator, or a survey. These approaches will all be briefly discussed. First, the focus groups: Kondyli and Elefteriadou [16] performed a study on merging behavior at freeways using a focus group. Participants had to discuss and indicate their thinking process during a merging maneuver from an on-ramp to a freeway. Secondly, the use of an instrumented vehicle: in Van Koningsbruggen and Stranner [17] the driving behavior at merging areas was studied using an instrumented vehicle. In this case the drivers commented in real-time on their behavior. This may lead to a driving behavior which might not reflect reality properly, since, due to the requirement of commenting in real-time, the drivers might make decisions more consciously. Thirdly, the use of driving simulator: other studies apply driving simulators to investigate the driving behavior [18]. Driving simulators are attractive to researchers, since they can be used to create a goal-oriented virtual world for implementing different traffic situations which cannot be tested easily in the real world. However, a potential disadvantage of driving simulators could be that they only provide a representation of reality, not reality itself. Finally, the use of surveys: several studies utilized (static) surveys (also called questionnaires, which we consider the same here) to investigate different aspects of driving behavior, for example, De Winter and Dodou [19] and Davey et al. [20]. The survey used the most is the Driver Behavior Questionnaire (DBQ) [21], which has been used many times, for example, by Bener et al. [22], Bener et al. [23], De Winter and Dodou [19], and Li et al. [24].

Most surveys require people to envisage the situation which might be an extra hurdle. For example, the study by De Craen et al. [25] used pictures of traffic situations to identify novice, unsafe, and overconfident drivers. Although many forms of the DBQ exist, a questionnaire using video clips is not common practice. In some other fields, in particular traffic safety and accident analysis, video clip based traffic surveys have recently been pioneered, for instance, for cyclist safety [26], or for the effect of visibility of work zones on the speed choice of car drivers [27]. But a questionnaire with video clips of particular traffic situations has never, to the best of the authors' knowledge, been used to ask people about their actions concerning speed and lane choice. Applying video clips can help to overcome the shortcoming of surveys that the situation is difficult to envisage. Though, the researchers have to be aware that drivers can have different driving behavior than shown in the video, because they feel they are in a situation they usually do not face.

Since we would like to investigate the distribution of people over the 4-lane change strategies mentioned before, a large response is needed, which is infeasible by test drives or focus groups. Video seemed a good means to that end. We found a way to adapt the videos to the driving style
TABLE 1: Categorization of respondents into three branches.

\begin{tabular}{lcc}
\hline Desired speed & Category & Speed in video clip \\
\hline$<103 \mathrm{~km} / \mathrm{h}$ & "Slow" driver & $\sim 100 \mathrm{~km} / \mathrm{h}$ \\
$103-110 \mathrm{~km} / \mathrm{h}$ & "Average" driver & $\sim 107 \mathrm{~km} / \mathrm{h}$ \\
$>110 \mathrm{~km} / \mathrm{h}$ & "Fast" driver & $\sim 115 \mathrm{~km} / \mathrm{h}$ \\
\hline
\end{tabular}

which became visible from the earlier part of the survey. Thus, with these personalized surveys with videos, we maintain the core quality of reaching out to many people (online), while still benefiting from relevant questions. The survey was also validated, by letting several respondents to the survey also drive in an instrumented vehicle to validate their choices. Section 5 will show how the validation was executed.

\section{Survey Setup}

In this section, we describe the setup of the survey, starting with the design (Section 3.1), followed by the recruitment and characteristics of the respondents (Section 3.2), and finally the analyses in Section 3.3.

3.1. Survey Design. The survey starts with a picture of an empty three-lane freeway with a speed limit of $100 \mathrm{~km} / \mathrm{h}$. For this situation, it is asked which lane the respondent will choose and what his speed will be in this situation. Based on the answers, a personalized survey is presented to the respondents, in which the indicated speed of driving in the videos is related to his or her own quoted driving behavior. The categories used for this are given in Table 1.

The survey is set up to find out the driver's strategy to choose a lane using a series of questions containing videos. The videos are reworked to give respondents the idea that the situation is as close as possible to their normal driving behavior. That means that a rear view mirror is added showing the video of a rear-facing camera. Also, a speed limit indication was added to the video clip. The speed shown as current speed is adapted to the desired speed of the respondent. Figure 1 shows a screenshot of the resulting video clip. In all video clips, a view of the traffic situation from the driver's perspective is given, and respondents were asked what they would do if they were in that vehicle.

The main research themes of the survey are to determine the following.

Theme 1. Does a respondent drive according to strategy 1,2 , or 3 ?

Theme 2. Does a respondent drive according to strategy 4 ?

Theme 3. Does a respondent strictly obey the keep right rule?

Theme 4. Would a respondent overtake another driver at the right side?

Theme 5. Would a respondent make a courtesy lane change to create space for a merging vehicle? 


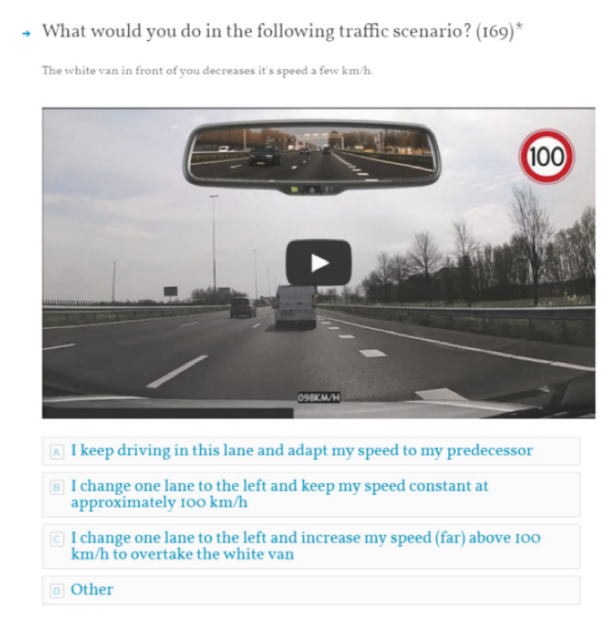

FIGURE 1: Screenshot of a video clip.

Based on these themes, 14 questions with corresponding video clips were formulated, which are reported in Table 2. In most cases, an answer would lead to a single strategy, since, apart from the action, also the motivation for the action was asked. In some cases, an answer might exclude some strategies, but not point at a single strategy. Some of the traffic situations in the different questions are similar, which is to test the consistency of the respondents. The videos can be downloaded [28]; note that they are available for reuse. Apart from the driving-strategy related questions, also the basic characteristics of the respondents such as age and yearly mileage were asked. On average, the survey took 17 minutes to complete.

3.2. Respondents. The survey was distributed via personal network of the researchers. More importantly, it has been distributed via communication of the Royal Dutch Touring Club (ANWB). Generally, the social media and e-mail newsletter of this organization are distributed to millions of Dutch motorists. To stimulate people to participate, a gift card of 50 euros was promised as a prize awarded to one randomly chosen respondent. In total, 1,258 respondents completed the survey. As demonstrated in Figure 2, the resulting group is not homogeneously distributed over ages. There is a peak for younger drivers (20-25 years old), possibly due to university involvement, and a peak for more senior drivers (over 60 years old).

3.3. Analyses. The analyses are structured along the 5 themes. For each theme, it is checked which fraction of respondents chooses the particular strategy in the applicable question.

The population of the respondents could cause a bias in the survey, because driving behavior is influenced by personal characteristics, training, and experience; see Fuller [29]. A particular subgroup that shows deviating driving behavior will affect the results if this subgroup is overrepresented in the sample. Hence, we would like to know the dependencies. The two most obvious dependencies have been tested: (1) the relation between desired speed at one hand and age and

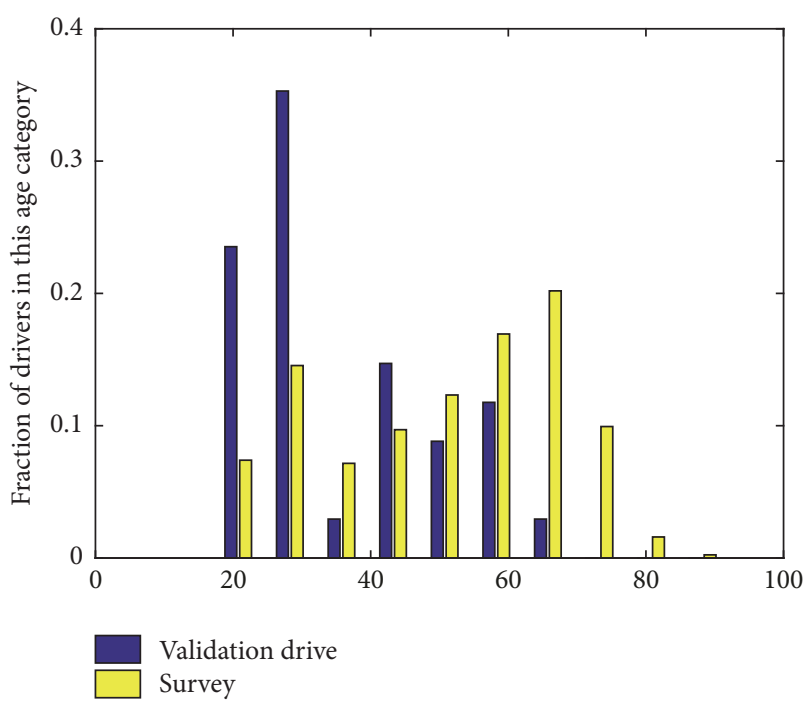

Figure 2: Age distribution of the respondents in the survey and participants in the drive.

experience at the other and (2) the relation between desired speed at one hand and frequency of driving and weekly mileage at the other. In Section 4.3 they are presented in pairs, since they are most correlated. Significance is tested with Cramer's V test.

\section{Survey Results}

This section describes the results. First, it discussed how often respondents choose a particular lane change strategy. Then, Section 4.2 presents results on overtaking at the right, and finally, Section 4.3 presents the desired speed of the respondents.

4.1. Lane Change Strategy Choice. Table 3 shows how the responses of the survey led to a distribution over strategies. Not all questions lead to the same distribution of respondents over the categories, which is in line with the effect seen in the drive. We also see that drivers change strategies for some questions. Nevertheless, we can derive a tendency: Strategy 1 is chosen most often, then strategy 3 , then strategy 2 , then strategy 4 . The lower part of Table 3 shows that strategy 1 is used by almost every respondent and also strategy 3 is used by $74 \%$ of the respondents. Strategy 2 is used by just over half of the drivers and strategy 4 is rarely used.

Table 4 shows to which extent respondents are keeping the right lane. Approximately $75 \%$ of the respondents would normally go back to the right lane if there is a possibility. Only for question 7, this fraction is considerably lower than $75 \%$. This is likely due to the fact that another vehicle is present in the center lane. Drivers do not prefer to switch lanes frequently.

4.2. Overtaking at the Right and Cooperating to Other Lane Changes. The vast majority of the respondents indicated that they would not overtake at the right; see Table 4 . This varied 
TABLE 2: Overview of the questions: screenshot (all but the first row cropped due to space limitation), question number, theme of question (in bold), and description.

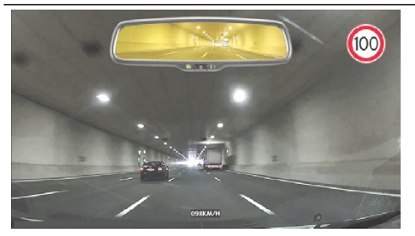

1: Right overtaking. You are driving in a tunnel on a 4-lane freeway on the third lane from the left. A truck is driving up ahead in the rightmost lane and a person car is driving in the second lane from the left while there is space for that driver to keep right using your lane. You are getting closer to that person car.

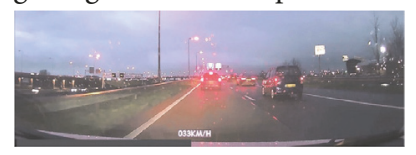

4: Strategies 1, 2, and 3. You are driving on a 2-lane freeway during congestion in the left lane. You are approaching the tail of a stop-and-go wave, while traffic in the right lane drives smoother and with a higher speed.

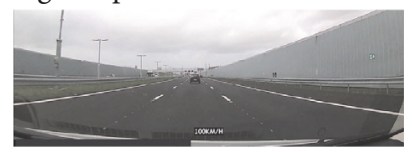

7: Keep right/Strategy 4. You are driving on a 3-lane freeway in the center lane while having passed a truck in the right lane. There is space to change lanes to the right after you have passed the truck, while a faster driver is approaching you from behind.

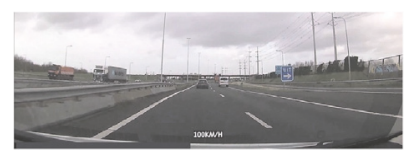

10: Keep right. You are driving on a 2-lane freeway in the left lane, while you pass a truck that is driving in the right lane. Up ahead another truck is driving there, while a faster driver is approaching you from behind.

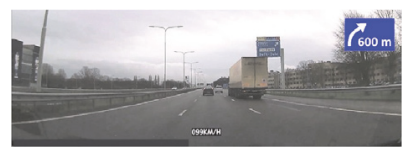

13: Strategies 1, 2, and 3. You are driving on a 3-lane freeway in the center lane. To follow your route you have to take the exit that starts at 600 meters from the moment the video stops. On that moment a truck is driving ahead in the right lane.

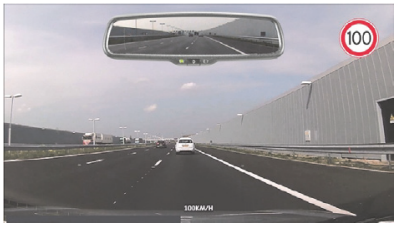

2: Strategies 1, 2, and 3. You are driving on a 3-lane freeway in the right lane. A slightly slower predecessor is driving in front of you and you are approaching that vehicle, while a black car just passed you with high speed.

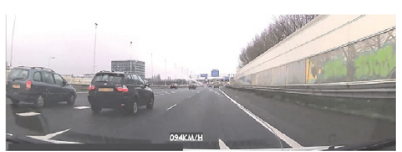

5: Right overtaking. You just entered the freeway via an on-ramp, while in the lane to the left of you a black car is driving with a low speed. You need to proceed on this carriageway to follow your route.

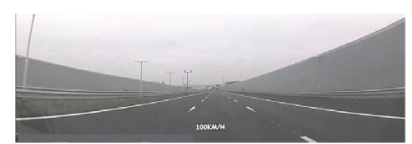

8: Keep right. You are driving on a 3-lane freeway in the center lane, while passing two trucks in the right lane. After you have passed the trucks there is an empty road ahead of you.

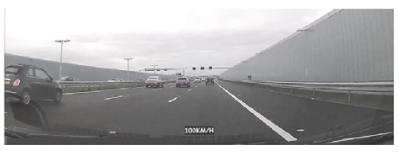

11: Strategy 4. You are driving on a 3-lane freeway in the rightmost lane with a speed of $100 \mathrm{~km} / \mathrm{h}$. However, all other vehicles around you drive much faster and you are overtaken by them.

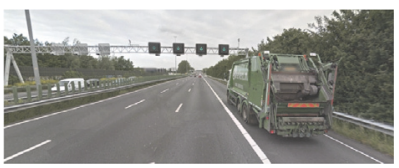

14: Keep right. (Static picture). You are driving on a 3-lane freeway in the rightmost normal lane. This road section is equipped with a rush-hour lane which is currently open for traffic. You will pass the truck that drives on the rush-hour lane.

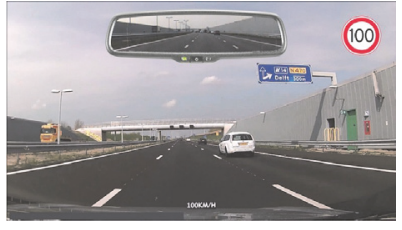

3: Strategies 1, 2, and 3. You are overtaking the white car, which increases its speed a bit, resulting in a very slow overtaking maneuver (this scenario is only shown to the respondents who want to overtake the vehicle with a constant speed).

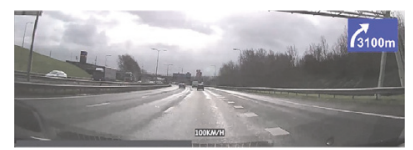

6: Strategies 1, 2, and 3. You merge onto the main road from an on-ramp, while to continue your route you need to take the second exit from that point which is 3100 meters up ahead.

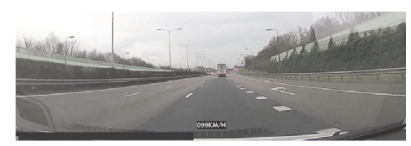

9: Strategies 1, 2, and 3. You are driving on a 3-lane freeway in the rightmost lane. A truck is driving in front of you in the same lane, while a faster driver is approaching you from behind on the center lane.

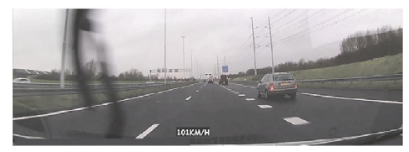

12: Courtesy lane change. You are driving on a 2-lane freeway in the right lane, while you approach a merging lane on which a vehicle is driving. However, the driver is not using its blinker. 
TABLE 3: Distribution of respondents over the lane change strategies.

\begin{tabular}{|c|c|c|c|c|c|c|c|c|c|}
\hline & & \multicolumn{7}{|c|}{ Lane change strategy } & \multirow{2}{*}{ Other answers } \\
\hline & & 1 & 2 & 3 & 4 & $1 / 2$ & $3 / 4$ & $3 *$ & \\
\hline \multicolumn{10}{|c|}{ Responses per question } \\
\hline Question & Theme & & & & & & & & \\
\hline 2 & Strategies 1,2 , and 3 & $77 \%$ & $10 \%$ & $12 \%$ & $1 \%$ & - & - & - & $0 \%$ \\
\hline 3 & Strategies 1,2 , and 3 & $56 \%$ & $43 \%$ & - & - & - & - & - & $1 \%$ \\
\hline 4 & Strategies 1,2 , and 3 & - & - & - & - & $27 \%$ & $73 \%$ & - & $0 \%$ \\
\hline 6 & Strategies 1,2 , and 3 & - & - & $44 \%$ & - & $28 \%$ & - & $27 \%$ & $1 \%$ \\
\hline 9 & Strategies 1,2 , and 3 & $85 \%$ & $11 \%$ & $3 \%$ & - & - & - & - & $1 \%$ \\
\hline 11 & Strategy 4 & - & - & - & $12 \%$ & $87 \%$ & - & - & $1 \%$ \\
\hline 13 & Strategies 1,2 , and 3 & $20 \%$ & $19 \%$ & $61 \%$ & - & - & - & - & $1 \%$ \\
\hline \multicolumn{10}{|c|}{ Cumulative use of strategies } \\
\hline \multicolumn{2}{|c|}{ Number of respondents } & 1211 & 665 & 931 & 11 & 1220 & 336 & 341 & \\
\hline \multicolumn{2}{|c|}{ Percentage of respondents } & $96 \%$ & $53 \%$ & $74 \%$ & $1 \%$ & $97 \%$ & $27 \%$ & 27 & \\
\hline
\end{tabular}

*: Question 6: drivers were instructed to exit in 3200 meters.

TABLE 4: Distribution of respondents to behavior characteristics.

\begin{tabular}{|c|c|c|c|c|}
\hline \multirow{2}{*}{ Question } & \multirow{2}{*}{ Theme } & & \multicolumn{2}{|c|}{ Changing to rightmost lane? } \\
\hline & & & Yes & No \\
\hline 7 & $\begin{array}{c}\text { Keep } \\
\text { right/Strategy } \\
4\end{array}$ & & $57 \%$ & $42 \%$ \\
\hline 8 & Keep right & & $88 \%$ & $12 \%$ \\
\hline 10 & Keep right & & $76 \%$ & $23 \%$ \\
\hline \multirow[t]{3}{*}{14} & Keep right & & $73 \%$ & $25 \%$ \\
\hline & & & \multicolumn{2}{|c|}{ Right overtaking? } \\
\hline & & & Yes & No \\
\hline 1 & $\begin{array}{c}\text { Right } \\
\text { overtaking }\end{array}$ & & $20 \%$ & $80 \%$ \\
\hline \multirow[t]{4}{*}{5} & $\begin{array}{c}\text { Right } \\
\text { overtaking }\end{array}$ & & $16 \%$ & $84 \%$ \\
\hline & & & \multicolumn{2}{|c|}{ Cooperative behavior? } \\
\hline & & & Yes & No \\
\hline & & Lane change & Speed adjustment & \\
\hline 12 & $\begin{array}{c}\text { Courtesy lane } \\
\text { change }\end{array}$ & $72 \%$ & $11 \%$ & $16 \%$ \\
\hline
\end{tabular}

for the two different questions on this topic: in question 1, $20 \%$ would overtake at the right, and in question $5,16 \%$ would overtake at the right.

Table 4 also shows the cooperative behavior. If another driver indicates the desire to change lane (e.g., the on-ramp lane ends, blinker on), $83 \%$ of the drivers will facilitate this lane change. Most respondents will do so by changing one lane to the left. Others (11\%) will reduce speed to allow the vehicle to merge in front.

4.3. Desired Speed. Figure 3 shows the quoted desired speeds of the respondents on the $100 \mathrm{~km} / \mathrm{h}$ section. For illustration purposes in the figure, we added a $3.75 \mathrm{~km} / \mathrm{h}$ margin (positive and negative). If this was omitted, the figure would have some

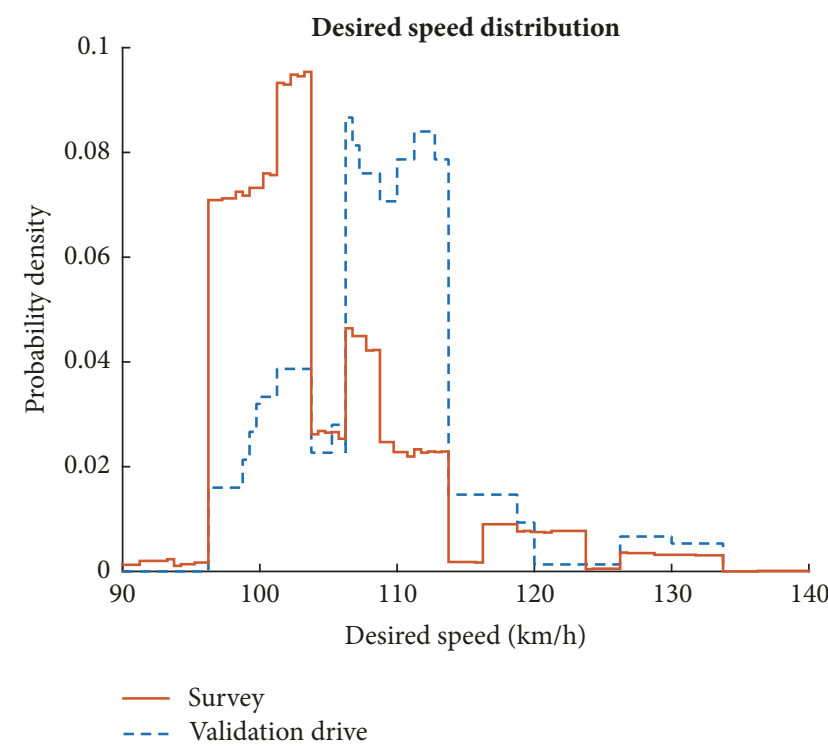

FIGURE 3: Distribution of quoted desired speeds at a road of a 100 $\mathrm{km} / \mathrm{h}$ speed limit.

spikes. The desired speed is on average $104.5 \mathrm{~km} / \mathrm{h}$, with a standard deviation of $8.0 \mathrm{~km} / \mathrm{h}$. This is hence slightly above the speed limit of $100 \mathrm{~km} / \mathrm{h}$.

The correlations with other factors are shown in Figure 4. We find that (1) younger and inexperienced drivers have a higher desired speed than older drivers (statistically significant and strong) and (2) frequent drivers and those with a higher weekly mileage have a higher desired speed than infrequent drivers (statistically significant and weak).

\section{Survey Validation}

The validation of the survey was done via drives. From the people who filled out the survey, 25 participants also drove an instrumented vehicle. The drive was recorded on video, 


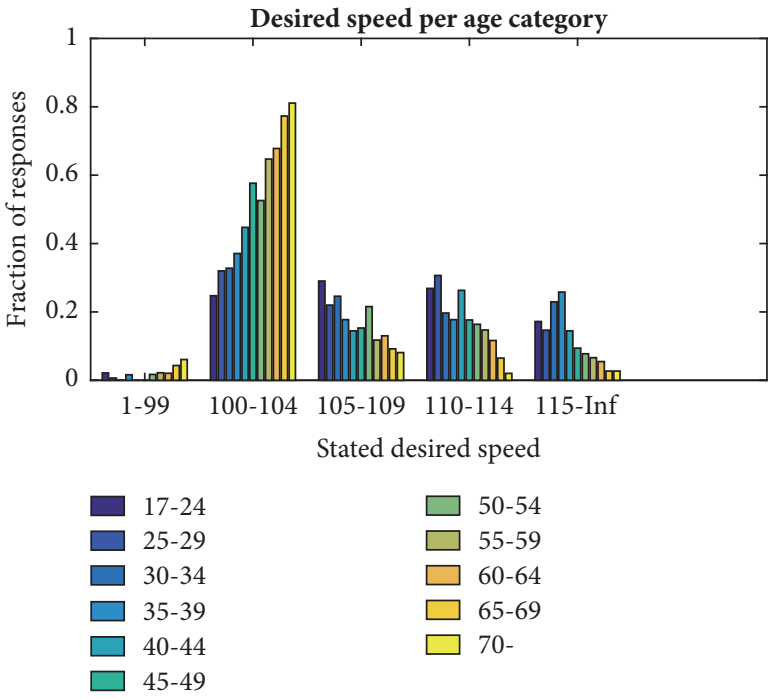

(a) Age

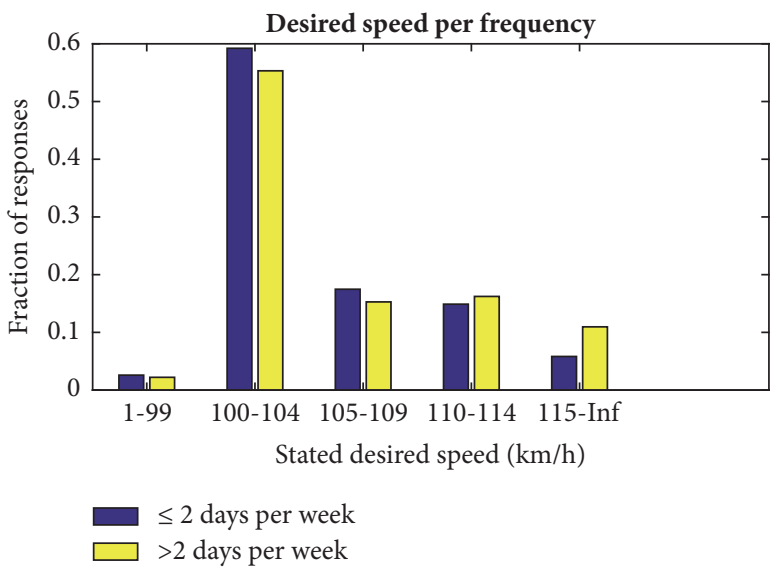

(c) Driving frequency

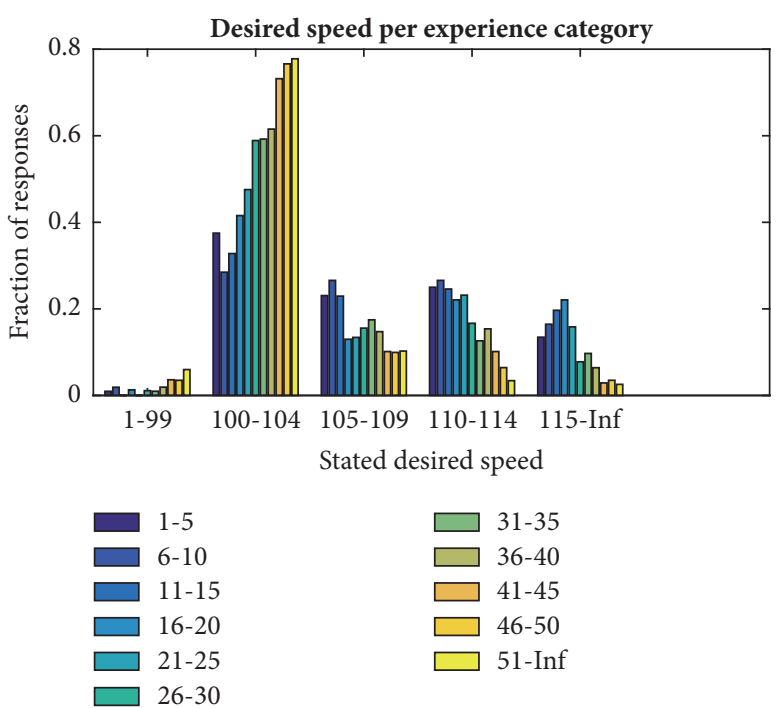

(b) Experience

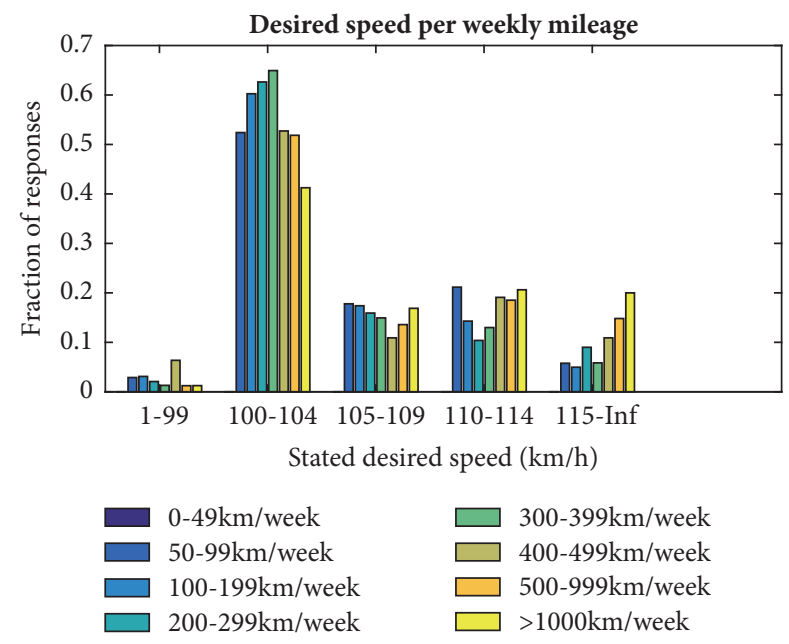

(d) Mileage

FIGURE 4: Overview of the impacts of various factors to desired speed.

which has been used to discuss the trip with the participants afterwards. Chronologically, the drives took place before the survey. This means that situations from the drives could be used in the survey, and the choice was recorded for the same person-once as participant and once as respondent. With the information from both sources, the validation took place in three stages.

First, the strategy/strategies of a participant was compared with the answers he gave in the survey. For each participant, it can be checked whether the answers given in the survey are in line with the strategy shown during the drive. For several questions it has been analyzed whether the strategy in the survey matches the profile as found during the drive. The answers to 6 questions (i.e., questions 2, 3, $4,6,9$, and 13) were analyzed and checked whether the chosen strategies match the set of strategies found during the drive. Besides this, it has been investigated whether the respondents who answered that they would follow strategy 4 in question 11 also have strategy 4 in the set of strategies applied during the drive. Unfortunately, strategy 4 has many different appearances and only 1 question explicitly referred to strategy 4 . So, even if the results of the drive in the instrumented vehicle and the survey do not match, results from the survey might still hold.

Second, we can identify specific videos to situations that an individual driver encountered and check to which extent the stated action is the same as the revealed action in this situation. This is limited to a maximum of one driver per question in the survey.

Third, the desired speed is compared for the drivers. This can be done for the drivers individually, as well as for the whole population, linked to the drivers characteristics.

The remainder of this section first discusses the setup of the drives and then discusses the validation results. 
5.1. Setup of Drives. For the drives and interviews, participants were recruited using the personal and professional networks of the researchers involved. The professional networks included people from the university, the Dutch main road authority (Rijkswaterstaat), and the local gardening center (part-time job of the student). These networks were employed as the base starting point, leading to a wider variety of participants. As a compensation for their contribution, all participants received a 25-euro gift card. In total, a group of 25 people both participated in the drive and took part in the survey. During their trip, three cameras were installed in the vehicle: one pointing forwards, one backwards, and one at the driver.

The drives took place in a period of 7 days, between 1 and 10 February 2016, during which the weather conditions were normal and did not vary much. The $40 \mathrm{~km}$ route for the drive includes the freeways A13, A20, and A4 in the east, south, and north direction, respectively. The freeways have different speed limits, various number of lanes, and speed enforcement (by police patrol, permanent and temporary speed cameras, and average speed check). Most participants come from the area and are familiar with the main freeways. A part of the route (the A4 stretch) was opened less than a month before the drives took place, which means most participants were unfamiliar with that road stretch. Ideally, people with different levels of route familiarity would be needed to participate in the drive.

Before the drive, drivers were instructed on the use of the vehicle; this was a normally operated vehicle, a Toyota Prius. The instrumentation of the car was not intrusive, nor limiting the operation of the car in any way. The car was equipped with an automatic gearbox (less common in the Netherlands than a manual gearbox). The drivers were informed about the route, and, in addition to that, if preferred by the participant, a hand-held navigation device was programmed to guide them. The drivers were instructed to drive safely and to drive as they normally would. During the drive, participants were alone in the car.

After the drive, an interview was carried out. This interview was semi-structured and consisted of two parts: one general part related to the driving style in general and another part concerning the particular drive of the participant. During the interviews, the four strategies were discussed with the drivers. They were asked which strategies they have been applying throughout the trip and which they would apply in other conditions. From this interview, their considerations and strategy were derived.

5.2. Validation Results. This section describes the validation results: first the chosen strategies of the participants and then the choice in the identical situation as part of the survey. Finally, the desired speed is compared.

5.2.1. Strategies per Participant. In the survey, all 25 participants are requested to choose 6 times between strategies 1,2 , and 3. Accounting for 7 missing or other answers, that gives $6 \times 25-7=143$ strategies. Moreover, 4 out of these 25 respondents answered to use strategy 4 in question 11 . Hence, in total, we have $143+4=147$ chosen strategies, which we can compare with the set of applied strategies found in the drive. For the majority of these chosen strategies (127 choices, or $86 \%)$ the given response is in line with one of the strategies derived from the drive, and so the rest (20 choices, or $14 \%$ ) is not in line. This has been built up as follows: 1 respondent indicated a different strategy in 4 questions; 1 respondent indicated a different strategy in 3 questions and the same strategy in 1 question; 2 respondents indicated a different strategy in 2 questions and the same strategy in 2 other questions; 9 respondents indicated a different strategy in 1 question and the same strategy in 3 questions. This means that 12 respondents indicated all questions in line with the strategies from the drive. With this $86 \%$ match between choices in the survey and the choices of the participants in the experimental drive, we can conclude that the survey is well in line with the results shown in the drive and can therefore be used as representative for real driving behavior.

5.2.2. Choice in Identical Situation. For the second validation approach, using exactly the same traffic situation for exactly the same driver, 7 questions could be checked. In 4 out of the 7 cases, the stated preference (in the survey) was exactly the same as the behavior the participant showed during the drive. Given the number of options (approximately 5 per question), this is pretty consistent. However, since the drivers have been shown to have multiple strategies while driving (see Table 3), other approaches of coping with a specific situation did not come as a surprise.

5.2.3. Desired Speed. The desired speeds are given in Figure 3. For the participants, the mean desired speed is $108.0 \mathrm{~km} / \mathrm{h}$ with a standard deviation of $8 \mathrm{~km} / \mathrm{h}$. This is higher than the desired speed of the respondents of the survey $(104.5 \mathrm{~km} / \mathrm{h})$. This might be due to the age of the participants. Figure 4 shows how the desired speed depends on the age. Section 4.3 showed that the desired speed is lower for higher ages, and the age for the respondents was significantly higher than for the participants in the test drive (see also Figure 2).

\section{Lane Changing in Other Countries}

To put the results in the perspective, a comparison has been made. The same survey has been distributed to drivers in two other countries: Switzerland and the United States of America (USA). This section describes the setup and results of this comparison.

6.1. Setup of the International Comparison. In Switzerland, traffic regulations are comparable to the Netherlands. The main difference is how these laws are enforced. Switzerland is known for their high traffic fines in case of noncompliance. Also, road design aspects are different. For example, the Swiss guidelines show a shorter length of the deceleration lane (150$170 \mathrm{~m}$ ) than the Dutch guidelines $(250 \mathrm{~m})$.

The rules in the United States are more different. Federal laws do not prohibit overtaking at the right on multilane freeways; however, some states have this prohibition, or a 
TABLE 5: Comparison of responses of Dutch and Swiss drivers for scenarios 3, 5, and 13.

\begin{tabular}{ccccc}
\hline \multirow{2}{*}{$\mathrm{Nr}$} & \multirow{2}{*}{ Question } & \multicolumn{2}{c}{ Nationality } \\
& & Response & Dutch & Swiss \\
\hline 3 & If you overtake a slower predecessor (passenger car), would you do & Strategy 1 & $56 \%$ & $73 \%$ \\
& this according to Strategy 1 or 2? & Strategy 2 & $43 \%$ & $27 \%$ \\
5 & Would you overtake another vehicle via its right side, when there is & Yes & $16 \%$ & $7 \%$ \\
& no possibility to overtake via the left side? & No & $84 \%$ & $93 \%$ \\
& & Yes, with constant speed & $20 \%$ & $12 \%$ \\
13 & If you have to take the exit 600 metres up ahead, would you & Yes, with speed increase & $19 \%$ & $3 \%$ \\
& overtake a truck just before the off-ramp? & No & $61 \%$ & $85 \%$ \\
\hline
\end{tabular}

prohibition of driving left for a prolonged period of time without overtaking. For an overview of the regulations in different states, see [30].

For the comparison, first the desired speeds are tested for differences. The independent sample $t$-test cannot be used for this since this test assumes that the dependent variable is normally distributed. Therefore, the nonparametric Mann-Whitney test is used to check whether there are differences in the medians of the desired speeds between (1) the Dutch and Swiss drivers, and (2) the Dutch and the American drivers.

Since the goal of the international survey is to identify differences, a smaller sample suffices. To find differences in resulting behavior, some answers of respondents can be grouped. For example, in scenario 10 the four multiple choice answers have been aggregated to two categories, namely, keep right behavior and keep lane behavior. This increases the statistical power of the survey, while at the same time justifying the goal of finding differences in revealed behavior. Using a Chi-square test, statistical significance was tested for confidence level of $95 \%$ and a confidence interval of $5 \%$. Important to note is that besides the statistical correlation, also the association is measured. The first indicates how unlikely it is that the result could be obtained by chance; the second one indicates how strong the relation is. To this end, the $\phi$ value of Cramer's $V$ test is used, with values under 0.1 being considered negligible [31].

The survey has been spread in Switzerland and the USA via the academic network. In total, the survey has been completed by 59 Swiss respondents and 40 respondents from the USA.

6.2. Results: Small Differences. It was found that the desired speed was not significantly different in Switzerland compared to the Netherlands. Mean speeds are close to each other: the Netherlands $104.5 \mathrm{~km} / \mathrm{h}$ and Switzerland $104.1 \mathrm{~km}$.h. The results of the test show no significant difference in the median values $(U=36, z=0.208, p=0.835)$. In the $U S A$, a higher speed was found: $109.1 \mathrm{~km} / \mathrm{h}$ as average. Also the median speed was considerably and significantly higher: 110 in the USA versus $100 \mathrm{~km} / \mathrm{h}$ in the Netherlands (Mann-Whitney test results: $\mathrm{U}=33, \mathrm{z}=4.055, \mathrm{p}=0.0005$ ).

Then the lane choice strategy was considered. With the clustered answers, most questions led to statistically significant different fraction of people choosing a lane change strategy. However, all differences found, even though statistically significant, are negligible in size, according to Cramer's $\mathrm{V}$ test.

Table 5 presents an overview of the unclustered responses that showed a small size difference for Dutch and Swiss drivers. The results show that Swiss drivers tend to increase their speed less often when they overtake a slower predecessor in comparison with Dutch drivers. Furthermore, less Swiss drivers overtake another vehicle on the right side than Dutch drivers. This is observed in both scenarios that concerned right overtaking behavior, although in only one scenario this difference was found significantly different by the Chi-square test. Furthermore, only $15 \%$ of the Swiss respondents would overtake the truck just before the off-ramp, while $39 \%$ of the Dutch respondents would do so. This results could be due to the shorter ramps in Switzerland.

For American drivers, more clear differences exist concerning the lane choice. Figure 5 shows the most relevant examples. In short, US drivers are less bound to go to the right and have a higher tendency to overtake at the right. This can be explained by the different legislation in the USA.

All in all, we conclude that, with similar legislation and similar conditions (European driving), similar driving behavior can be expected.

\section{Discussion and Conclusions}

In this paper, people's driving strategies on the freeway have been studied. A large scale survey has been carried out. In total 1,258 respondents have filled out an online survey containing video clips. The survey has been validated by drives in an instrumented vehicle. For 25 people the answers in the survey were compared with the choice of strategy in a real-world case. Apart from the general chosen strategy, also a few identical situations could be identified, which further validated the survey.

The survey showed that most people choose a speed first and adapt the lane based on it. Less often people would have a strategy with a desired speed, but change their desired speed during overtaking. Also the strategy to choose a lane and adapt the speed accordingly is chosen less often than the strategy with the fixed desired speed. Only a small fraction of the drivers would neither have a desired speed nor a 


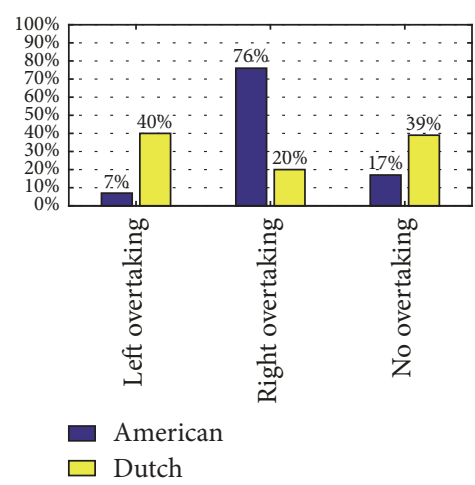

(a) Overtake slower driver that is unnecessarily driving in the second lane from the left in question 1

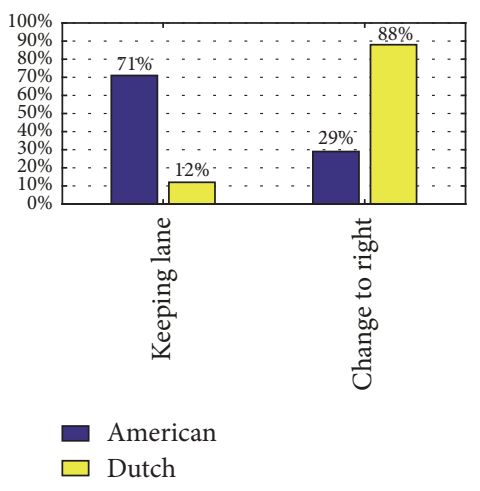

(b) Keep right on a 3-lane motorway

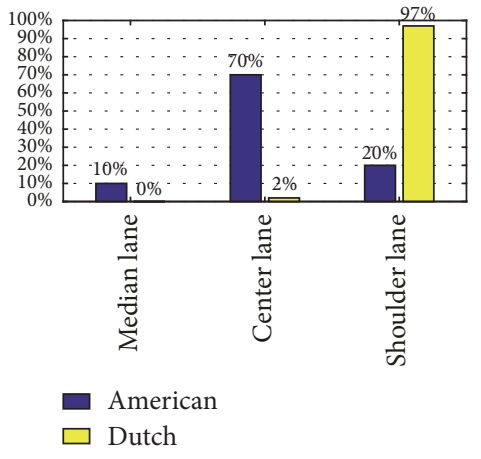

(c) Stated lane choice

FIGURE 5: Differences in lane choice for US drivers.

desired lane. A large number (75-80\%) of the respondents indicated that they would return to the right lane, and over $80 \%$ refrained from overtaking at the right.

In over $80 \%$ of the cases, drivers were willing to adapt their speed or (more frequently) their lane to facilitate a mandatory lane change of another driver. This courtesy behavior should definitely be incorporated into microscopic simulation models.

In addition, the paper investigated a desired speed distribution for freeways with a $100 \mathrm{~km} / \mathrm{h}$ speed limit. On average, the desired speed lies slightly above the speed limit, and younger drivers had a significantly higher desired speed. Moreover, drivers, who had higher mileage, had a higher desired speed than those who drive less; this effect is statistically significant, but very small in size.

The drives revealed that people have different strategies throughout their trip. For instance, they sometimes stick to a specific speed and adapt the lane accordingly, and sometimes they stick to a lane and adapt their speed. However, in general it can be concluded that there are various lane change and lane choice strategies. The research steps focus on developing the specific algorithms describing the decision-making in each of the strategies, and the effects thereof. These can then be implemented in microscopic simulation models. Preliminary progress steps have been published [32]. That work can now be expanded using the distributions found here.

The results presented here are valid for Dutch drivers. For Switzerland, with similar driving regulations, similar behavior was found. However, for the USA where the keepright-unless-overtaking rule does not apply as in Europe, behavior was found to be different. Percentages in each of the categories of behavior are therefore expected to be similar for Europe but will differ significantly for other continents. A similar study could reveal the driving behavior in these other continents. The setup presented in this paper can be used for this goal. Recommendation for future research hence includes performing this study for an international group of respondents and comparing driving strategies of drivers from various countries.
The current study contributes to a more realistic description of driving behavior and would hence improve the applicability of microscopic simulation tools for ex-ante traffic engineering changes. These tools describe how drivers will drive with a particular road layout. The resulting traffic patterns will follow from the microscopic interaction in the simulation. It would be interesting to assess how the effects of heterogeneity in lane change strategies work out for traffic operations. Also for assessing a new road design or new lane change or merging regulations, the insights from this paper could be used to improve microscopic traffic simulation models and to change them into powerful tools, also for these kinds of applications.

\section{Conflicts of Interest}

The authors declare that there are no conflicts of interest regarding the publication of this paper.

\section{Acknowledgments}

This research is supported by the Netherlands Organization of Scientific Research; the research has been conducted at the ITS Edulab, a cooperation between Rijkswaterstaat and Delft University of Technology.

\section{References}

[1] C. F. Daganzo, "A behavioral theory of multi-lane traffic flow. Part I: long homogeneous freeway sections," Transportation Research Part B: Methodological, vol. 36, no. 2, pp. 131-158, 2002.

[2] V. L. Knoop, A. Duret, C. Buisson, and B. Van Arem, "Lane distribution of traffic near merging zones influence of variable speed limits," in Proceedings of IEEE Intelligent Transportation Systems, 2010.

[3] S. Ahn and M. Cassidy, "Freeway traffic oscillations and vehicle lane-change," in Proceedigs of the International Symposium of Transportation and Traffic Theory, R. E. Allsop, M. G. H. Bell, 
and B. G. Heydecker, Eds., pp. 691-710, Elsevier, Amsterdam, the Netherlands, 2007.

[4] J. A. Laval and C. F. Daganzo, "Lane-changing in traffic streams," Transportation Research Part B: Methodological, vol. 40, no. 3, pp. 251-264, 2006.

[5] M. Keyvan-Ekbatani, V. L. Knoop, and W. Daamen, "Categorization of the lane change decision process on freeways," Transportation Research Part C: Emerging Technologies, vol. 69, pp. 515-526, 2016.

[6] V. Knoop, M. Keyvan-Ekbatani, M. De Baat, H. Taale, and S. Hoogendoorn, "Strategy-based driving behaviour on freeways: Findings of test-drive and on-line survey study," in Proceedings of the 96th Annual Meeting of the Transportation, 2017.

[7] T. Toledo, C. Choudhury, and M. Ben-Akiva, "Lane-changing model with explicit target lane choice," Transportation Research Record: Journal of the Transportation Research Board, pp. 157$165,1934$.

[8] A. Kesting, M. Treiber, and D. Helbing, "General lanechanging model MOBIL for car-following models," Transportation Research Record: Journal of the Transportation Research Board, vol. 1999, no. 1, pp. 86-94, 2007.

[9] S. Moridpour, M. Sarvi, and G. Rose, "Lane changing models: a critical review," Transportation Letters, vol. 2, no. 3, pp. 157-173, 2010.

[10] Z. Zheng, "Recent developments and research needs in modeling lane changing," Transportation Research Part B: Methodological, vol. 60, pp. 16-32, 2014.

[11] T. L. Pan, W. H. K. Lam, A. Sumalee, and R. X. Zhong, "Modeling the impacts of mandatory and discretionary lanechanging maneuvers," Transportation Research Part C: Emerging Technologies, vol. 68, pp. 403-424, 2016.

[12] J. A. Laval and L. Leclercq, "A mechanism to describe the formation and propagation of stop-and-go waves in congested freeway traffic," Philosophical Transactions of the Royal Society A: Mathematical, Physical \& Engineering Sciences, vol. 368, no. 1928, pp. 4519-4541, 2010.

[13] S. Ossen, S. Hoogendoorn, and B. G. H. Gorte, "Interdriver Differences in Car-Following: A Vehicle Trajectory-Based Study," Transportation Research Record: Journal of the Transportation Research Board, no. 1965, pp. 121-129, 2006.

[14] K. Ahmed, M. Ben-Akiva, H. Koutsopoulos, and R. Mishalani, "Models of freeway lane changing and gap acceptance behavior," in Transportation and Traffic Theory, vol. 13, pp. 501-515, 1996.

[15] W. J. Schakel, V. Knoop, and B. Van Arem, "Integrated lane change model with relaxation and synchronization," Transportation Research Record, vol. 2316, pp. 47-57, 2012.

[16] A. Kondyli and L. Elefteriadou, "Driver behavior at freewayramp merging areas: Focus group findings," Transportation Research Record: Journal of the Transportation Research Board, no. 2124, pp. 157-166, 2009.

[17] P. Van Koningsbruggen and T. Stranner, "EGNOS from an European Service Provider Perspective-Test results and Conclusions," Technical report, ERTICO, GNSS-enabled Services Convergence, 2011.

[18] J. C. F. de Winter, S. de Groot, M. Mulder, P. A. Wieringa, J. Dankelman, and J. A. Mulder, "Relationships between driving simulator performance and driving test results," Ergonomics, vol. 52, no. 2, pp. 137-153, 2009.

[19] J. C. F. De Winter and D. Dodou, "The driver behaviour questionnaire as a predictor of accidents: A meta-analysis," Journal of Safety Research, vol. 41, no. 6, pp. 463-470, 2010.
[20] J. Davey, D. Wishart, J. Freeman, and B. Watson, "An application of the driver behaviour questionnaire in an Australian organisational fleet setting," Transportation Research Part F: Traffic Psychology and Behaviour, vol. 10, no. 1, pp. 11-21, 2007.

[21] J. Reason, A. Manstead, S. Stephen, J. Baxter, and K. Campbell, "Errors and violations on the roads: A real distinction?" Ergonomics, vol. 33, no. 10-11, pp. 1315-1332, 1990.

[22] A. Bener, M. G. A. Al Maadid, T. Özkan, D. A. E. Al-Bast, K. N. Diyab, and T. Lajunen, "The impact of four-wheel drive on risky driver behaviours and road traffic accidents," Transportation Research Part F: Traffic Psychology and Behaviour, vol. 11, no. 5, pp. 324-333, 2008.

[23] A. Bener, T. Özkan, and T. Lajunen, "The Driver Behaviour Questionnaire in Arab Gulf countries: Qatar and United Arab Emirates," Accident Analysis \& Prevention, vol. 40, no. 4, pp. 1411-1417, 2008.

[24] J. Li, H. van Zuylen, and E. van der Horst, "The Driver Behaviour Questionnaire: An Investigation Study Applied to Chinese Drivers," in Computer-based Modelling and Optimization in Transportation, J. F. de Sousa and R. Rossi, Eds., vol. 262 of Advances in Intelligent Systems and Computing, pp. 433-447, Springer International Publishing, Cham, Switzerland, 2014.

[25] S. De Craen, D. A. Twisk, M. P. Hagenzieker, H. Elffers, and K. A. Brookhuis, "The development of a method to measure speed adaptation to traffic complexity: Identifying novice, unsafe, and overconfident drivers," Accident \& Analysis Prevention, vol. 40, no. 4, pp. 1524-1530, 2008.

[26] E. Lehtonen, V. Havia, A. Kovanen, M. Leminen, and E. Saure, "Evaluating bicyclists' risk perception using video clips: Comparison of frequent and infrequent city cyclists," Transportation Research Part F: Traffic Psychology and Behaviour, vol. 41, pp. 195-203, 2016.

[27] R. T. Steinbakk, P. Ulleberg, F. Sagberg, and K. I. Fostervold, "Analysing the influence of visible roadwork activity on drivers' speed choice at work zones using a video-based experiment," Transportation Research Part F: Traffic Psychology and Behaviour, vol. 44, pp. 53-62, 2017.

[28] M. De Baat and V. Knoop, "Movies lane changing," available at: http://doi.org/10.4121/uuid:771cdc12-ddd8-4607-8ec2bab096351824, 2018.

[29] R. Fuller, "Towards a general theory of driver behaviour," Accident Analysis \& Prevention, vol. 37, no. 3, pp. 461-472, 2005.

[30] M. I. O. T. MIT, “State "keep right” laws,” URL: http://www.mit .edu/jfc/right.html, Accessed: 26-07-201.

[31] L. M. Rea and R. A. Parker, Designing and conducting survey research: A comprehensive guide, John Wiley \& Sons, 2014.

[32] M. Keyvan Ekbatani, V. Grebert, W. Daamen, and V. L. Knoop, "Lane changing and speed interaction on freeways: An analytical microscopic study," in Proceedings of the 11th Traffic and Granular Flow Conference, TGF15, pp. 1-8, Springer, Nootdorp, The Netherlands, 2016. 


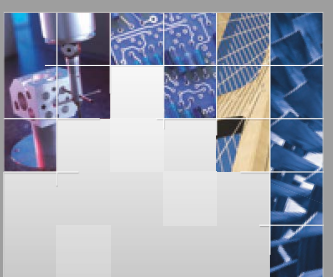

\section{Enfincering}
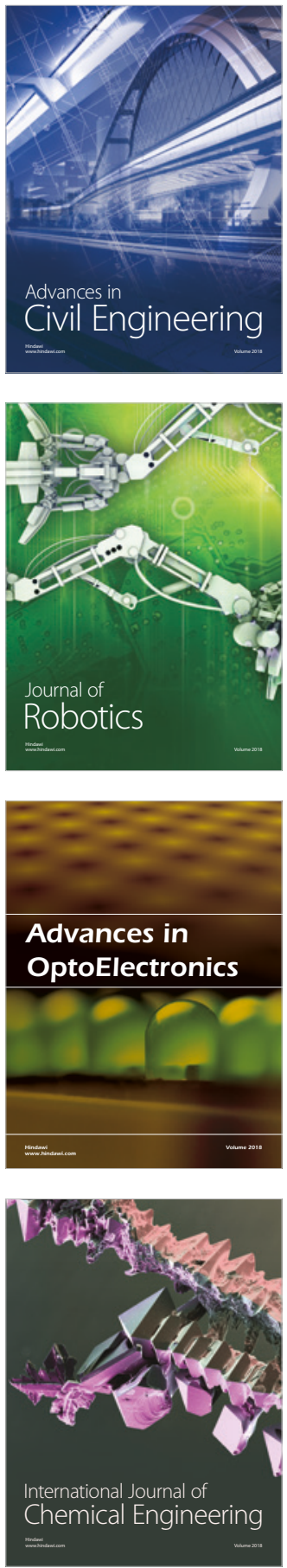

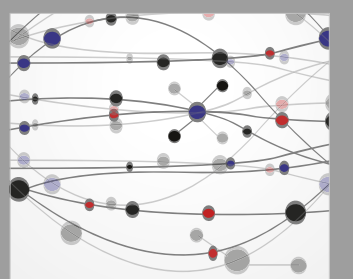

\section{Rotating \\ Machinery}

The Scientific World Journal

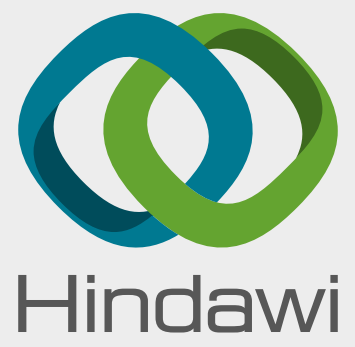

Submit your manuscripts at

www.hindawi.com
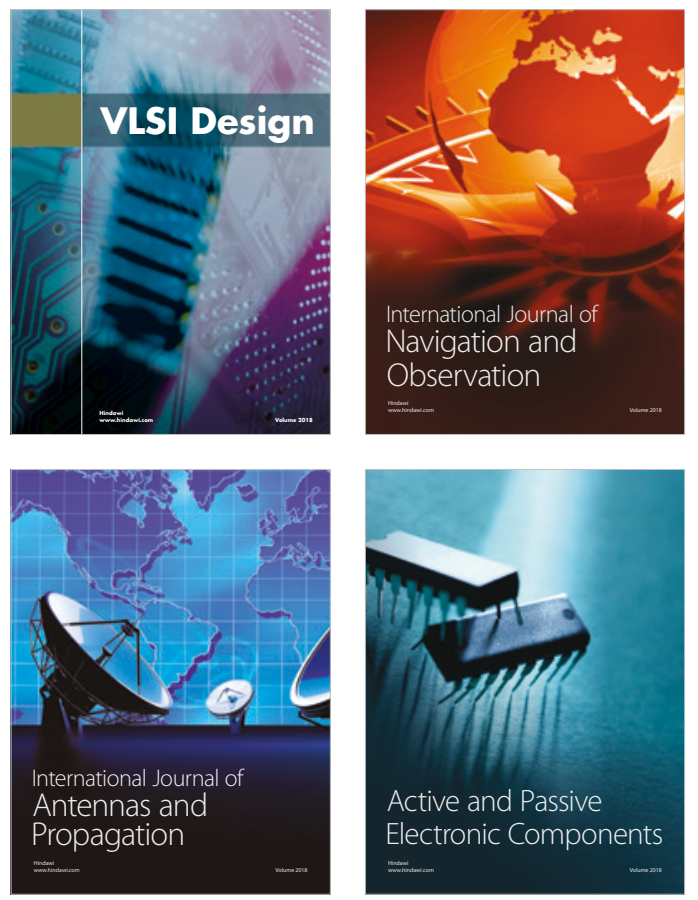
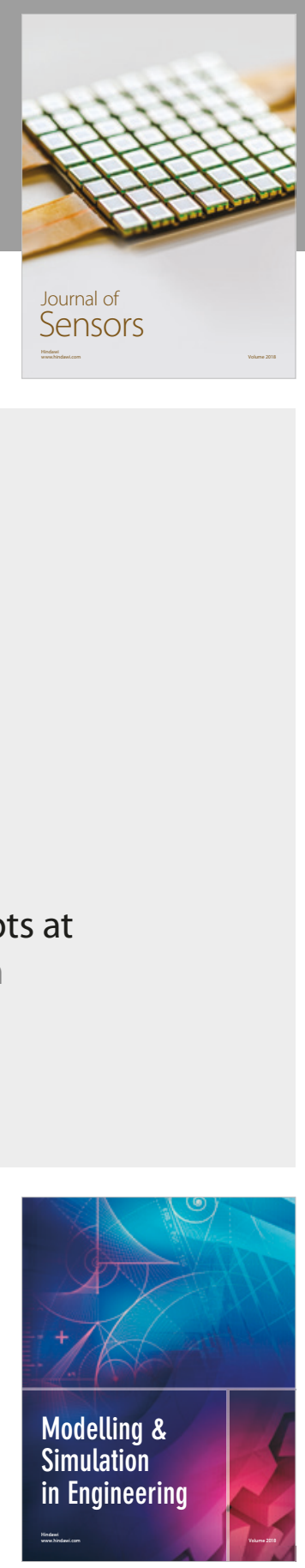

\section{Advances \\ Multimedia}
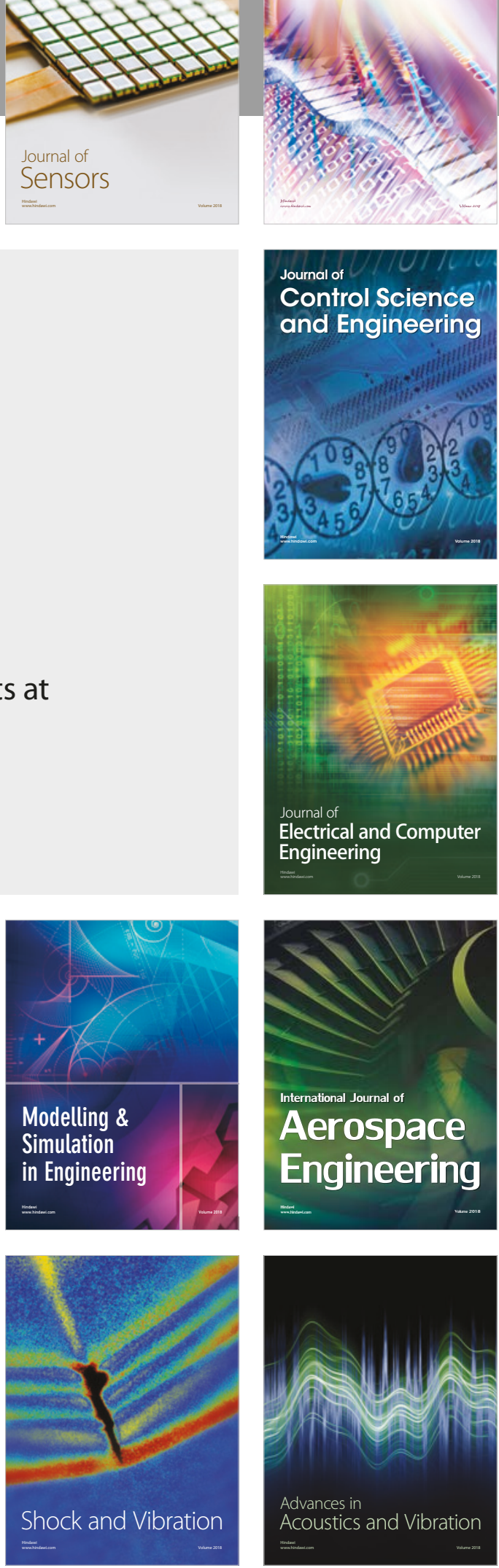